\title{
Addendum to: Multilevel dual approach for pricing American style derivatives
}

\author{
Denis Belomestny • Mark Joshi · John \\ Schoenmakers
}

Received: 24 March 2015 / Accepted: 04 May 2015

\begin{abstract}
In this note, we show how the dual approach in its particular form presented in [1] can be fitted into the framework of the recent work [2].
\end{abstract}

Keywords Optimal stopping, Dual approach, Multilevel Monte Carlo

Mathematics Subject Classification (2010) 91G60, 65C05, 60G40

JEL Classification G10, G12, G13

In the recent paper [2], a class of methodologies for developing upper bounds for Bermudan derivatives via Monte Carlo simulation is studied. This class, in particular, is designed to study methods that involve sub-simulations. Unfortunately, one of the most popular upper bound methodologies, that of Andersen and Broadie [1], does not lie within this class, since while the process used is an approximation to a martingale, the approximation itself is not a martingale with respect to some enlarged filtration as in Example 3.1 in [2].

We recall the dual approach, originally proposed by Rogers [4] and Haugh and Kogan [3], in the setup of [2]. Let

$$
\left(Z_{i}: i=0,1, \ldots, T\right), \quad T \in \mathbb{N}
$$

be a discrete-time, nonnegative stochastic process on a filtered probability space $(\Omega, \mathcal{F}, \mathbb{F}, \mathbb{P})$ and adapted to the filtration $\mathbb{F}:=\left(\mathcal{F}_{i}: 0 \leq i \leq T\right)$. It is assumed that

$$
\mathbb{E}\left[Z_{i}\right]<\infty \text { for } 0 \leq i \leq T .
$$

The problem is to find the optimal time to stop in order to maximize the value of $\left(Z_{i}\right)$. Let $\mathcal{T}_{i}$ denote the set of stopping times taking values in $\{i, i+1, \ldots, n\}$. A well-known

Duisburg-Essen University, Thea-Leymann-Str. 9, D-45127 Essen, Germany, and National Research University Higher School of Economics, Moscow, Russia, E-mail: denis.belomestny@uni-due.de · Centre for Actuarial Studies, Department of Economics, University of Melbourne VIC 3010, Australia, E-mail: mark.joshi@unimelb.edu.au · Weierstrass Institute for Applied Analysis and Stochastics, Mohrenstr. 39, D-10117 Berlin, E-mail: schoenma@wias-berlin.de 
fact is that the value of the optimal stopping problem is given by the Snell envelope

$$
Y_{i}^{*}=\sup _{\tau \in \mathcal{T}_{i}} \mathbb{E}_{i}\left[Z_{\tau}\right], \quad 0 \leq i \leq T,
$$

at time $i=0$, i.e., by $Y_{0}^{*}$. In the following, $\tau$ denotes a stopping time, $\mathbb{E}_{i}:=\mathbb{E}_{\mathcal{F}_{i}}$ denotes the conditional expectation with respect to the $\sigma$-algebra $\mathcal{F}_{i}$, and sup (inf) is to be understood as essential supremum (essential infimum).

The dual approach is based on the following observation: for any martingale $\left(M_{j}\right)$ with $M_{0}=0$, we have

$$
Y_{0}^{*}=\sup _{\tau \in \mathcal{T}_{0}} \mathbb{E}_{0}\left[Z_{\tau}\right]=\sup _{\tau \in \mathcal{T}_{0}} \mathbb{E}_{0}\left[Z_{\tau}-M_{\tau}\right] \leq \mathbb{E}_{0}\left[\max _{0 \leq j \leq T}\left(Z_{j}-M_{j}\right)\right],
$$

so that the right-hand side provides an upper bound for $Y_{0}^{*}$. In [3] and [4], it is shown that $(0.1)$ holds with equality (and the equality is almost sure) for the martingale part of the Doob decomposition of $Y^{*}$, that is,

$$
Y_{j}^{*}=Y_{0}^{*}+M_{j}^{*}-A_{j}^{*},
$$

where

$$
M_{j}^{*}=\sum_{i=1}^{j}\left(Y_{i}^{*}-\mathbb{E}_{i-1}\left[Y_{i}^{*}\right]\right), \quad A_{j}^{*}=\sum_{i=1}^{j}\left(Y_{i-1}^{*}-\mathbb{E}_{i-1}\left[Y_{i}^{*}\right]\right) .
$$

In practice, there is a variety of ways to implement the dual method of [4] and [3]. A straightforward way is to approximate the Doob martingale of the Snell envelope by using sub-simulations to estimate continuation values, for example due to a given approximate value function or due to a given suboptimal exercise strategy. These approaches naturally lead to an upper-biased estimate and one objective in [2] was to treat them in a unified way. To this end, Belomestny, Schoenmakers and Dickmann [2] choose a setup where the approximated martingales are martingales themselves with respect to some enlarged filtration, thus allowing direct application of the results of [4] and ensuring upper-biased estimates. Although various sub-simulation-based algorithms fall into this setup, the particular Andersen and Broadie [1] algorithm (based on suboptimal stopping families) does not.

Let us recap in more detail. First, a strategy is fixed. Since we have to consider values from forward starting points, this is a vector $\left(\tau_{j}\right)$ of stopping times with $\tau_{j} \geq j$ and

$$
\tau_{j}>j \Longrightarrow \tau_{j+1}=\tau_{j}
$$

Given these, we define a value process

$$
Y_{j}=\mathbb{E}_{j}\left[Z_{\tau_{j}}\right]
$$

[1] define a martingale analogously to $(0.2)$ by

$$
\begin{aligned}
M_{j}^{A B} & =\sum_{i=1}^{j}\left(Y_{i}-\mathbb{E}_{i-1}\left[Y_{i}\right]\right)=\mathbb{E}_{j}\left[Y_{j+1}\right]-Y_{0}+\sum_{i=0}^{j}\left(Y_{i}-\mathbb{E}_{i}\left[Y_{i+1}\right]\right) \\
& =\mathbb{E}_{j}\left[Y_{j+1}\right]-Y_{0}+\sum_{i=0}^{j}\left(Z_{i}-\mathbb{E}_{i}\left[Y_{i+1}\right]\right) 1_{\left\{\tau_{i}=i\right\}} .
\end{aligned}
$$


The expectations in this martingale are not immediate and have to be estimated via Monte Carlo simulation. The term $Y_{0}$ may be computed with high accuracy based on non-nested Monte Carlo simulations, and so $M_{j}^{A B}$ may be approximated by

$$
M_{j}^{A B, k}=\frac{1}{k} \sum_{\ell=1}^{k} \xi_{j+1}^{(\ell)}-Y_{0}+\sum_{i=0}^{j}\left(Z_{i}-\frac{1}{k} \sum_{\ell=1}^{k} \xi_{i+1}^{(\ell)}\right) 1_{\left\{\tau_{i}=i\right\}}
$$

where, just as in [2], the conditionally on $\mathcal{F}_{T}$ independent random variables $\xi_{j}^{(\ell)}$ are characterized by

$$
\mathbb{E}_{T}\left[\xi_{j}^{(\ell)}\right]=\mathbb{E}_{j-1}\left[\xi_{j}^{(\ell)}\right]=\mathbb{E}_{j-1}\left[Y_{j}\right]=\mathbb{E}_{j-1}\left[Z_{\tau_{j}}\right], \quad j=1, \ldots, T,
$$

and in particular it holds that

$$
\mathbb{E}_{j}\left[M_{j}^{A B, k}\right]=M_{j}^{A B}
$$

is a martingale with respect to the filtration $\mathbb{F}$.

The problem is that $\left(M_{j}^{A B, k}\right)$ is not a martingale with respect to the canonically enlarged filtration $\mathbb{F}^{\prime}:=\left(\mathcal{F}_{i}^{\prime}: 0 \leq i \leq T\right)$ with

$$
\mathcal{F}_{j}^{\prime}:=\mathcal{F}_{j} \vee \sigma\left(\xi_{p}^{(\ell)}, p=1, \ldots, j, \ell=1, \ldots, k\right),
$$

unlike the martingale (3.2) of Example 3.1 in [2], since (0.3) is even not adapted to $\mathbb{F}^{\prime}$. In fact, the adaptedness is destroyed by the presence of the indicator in $(0.3)$, that is, by the fact that in general $1_{\left\{\tau_{i}=i\right\}} \neq 1$ with positive probability.

The lack of the martingale property for the "true" algorithm from [1] is not taken into account in [2]; but it turns out that the full martingale condition is not necessary to obtain an upper bound. Consider equation $(0.1)$. There we assumed that $\left(M_{j}\right)$ is a martingale with respect to $\mathbb{F}$. We now show that the weaker condition that $\left(\mathbb{E}_{j}\left[M_{j}\right]\right)$ is a martingale with respect to the filtration $\mathbb{F}$ is sufficient (cf. $(0.4))$. Indeed, if $\left(M_{j}\right)$, with $M_{0}=0$, is adapted to some extended filtration such that $\left(\tilde{M}_{j}\right):=\left(\mathbb{E}_{j}\left[M_{j}\right]\right)$ is a martingale, we have

$$
\begin{gathered}
Y_{0}^{*}:=\sup _{\tau} \mathbb{E}\left[Z_{\tau}\right]=\sup _{\tau} \mathbb{E}\left[Z_{\tau}-\tilde{M}_{\tau}\right]=\sup _{\tau} \mathbb{E} \sum_{j=0}^{T} 1_{\{\tau=j\}}\left(Z_{j}-\mathbb{E}_{j}\left[M_{j}\right]\right) \\
=\sup _{\tau} \mathbb{E}\left[\sum_{j=0}^{T} \mathbb{E}_{j}\left[1_{\{\tau=j\}}\left(Z_{j}-M_{j}\right)\right]\right]=\sup _{\tau} \mathbb{E}\left[Z_{\tau}-M_{\tau}\right] \leq \mathbb{E}\left[\max _{j=0, \ldots, T}\left(Z_{j}-M_{j}\right)\right] .
\end{gathered}
$$

Under this extension of the framework, we can set $M_{j}=M_{j}^{A B, k}$, and then the algorithm from [1] is encompassed by the proofs of [2] and all the results go over. In particular, it then follows that the rate of convergence of the bias caused by subsimulations is as in that paper and a multi-level methodology can be implemented. 


\section{References}

1. L. Andersen and M. Broadie (2004). A primal-dual simulation algorithm for pricing multidimensional American options. Management Science, 50, 1222-1234.

2. D. Belomestny, J. Schoenmakers, and F. Dickmann (2013). Multilevel dual approach for pricing American style derivatives. Finance Stoch., 17, 717-742.

3. M. Haugh, L. Kogan (2004). Pricing American options: a duality approach. Operations Research, 52, $258-270$.

4. L.C.G. Rogers (2002). Monte Carlo valuation of American options. Mathematical Finance, 12, 271 286. 


\section{University Library}

\section{- M M I N E R VA A gateway to Melbourne's research publications}

Minerva Access is the Institutional Repository of The University of Melbourne

Author/s:

Belomestny, D;Joshi, M;Schoenmakers, J

Title:

Multilevel dual approach for pricing American style derivatives (vol 17, pg 717, 2013)

Date:

2015-07-01

Citation:

Belomestny, D., Joshi, M. \& Schoenmakers, J. (2015). Multilevel dual approach for pricing American style derivatives (vol 17, pg 717, 2013). FINANCE AND STOCHASTICS, 19 (3), pp.681-684. https://doi.org/10.1007/s00780-015-0267-x.

Persistent Link:

http://hdl.handle.net/11343/282883 